\title{
Inhibition of N-linked glycosylation impairs ALK phosphorylation and disrupts pro-survival signaling in neuroblastoma cell lines
}

Federica Del Grosso ${ }^{1}$, Marilena De Mariano ${ }^{1,2}$, Lorena Passoni ${ }^{3,5}$, Roberto Luksch³ ${ }^{3}$ Gian Paolo Tonini ${ }^{{ }^{*}}$ and Luca Longo ${ }^{1,4}$

\begin{abstract}
Background: The Anaplastic Lymphoma Kinase (ALK) is an orphan receptor tyrosine kinase, which undergoes posttranslational N-linked glycosylation. The catalytic domain of ALK was originally identified in the $t(2 ; 5)$ translocation that produces the unglycosylated oncogenic protein NPM-ALK, which occurs in Anaplastic Large Cell Lymphoma (ALCL). Recently, both germline and somatic activating missense mutations of ALK have been identified in neuroblastoma (NB), a pediatric cancer arising from neural crest cells. Moreover, we previously reported that ALK expression is significantly upregulated in advanced/metastatic NB. We hypothesized that ALK function may depend on N-linked glycosylation and that disruption of this post-translational modification would impair ALK activation, regardless the presence of either gene mutations or overexpression.
\end{abstract}

Methods: We employed tunicamycin to inhibit N-linked glycosylation. The following ALK-positive NB cell lines were used: SH-SY5Y and KELLY (ALK mutation F1174L), UKF-NB3 (ALK mutation R1275Q) and NB1 (ALK amplification). As a control, we used the NB cell lines LA1-5S and NB5 (no ALK expression), and the ALCL cell line SU-DHL1 (NPM-ALK).

Results: Tunicamycin treatment of ALK-positive NB cells resulted in a hypoglycosylated ALK band and in decreased amounts of mature full size receptor. Concomitantly, we observed a marked reduction of mature ALK phosphorylation. On the contrary, tunicamycin had no effects on NPM-ALK phosphorylation in SU-DHL1 cells. Moreover, phosphorylation levels of ALK downstream effectors (AKT, ERK1/2, STAT3) were clearly impaired only in ALK mutated/amplified NB cell lines, whereas no significant reduction was observed in both ALK-negative and NPM-ALK-positive cell lines. Furthermore, inhibition of N-linked glycosylation considerably impaired cell viability only of ALK mutated/amplified NB cells. Finally, the cleavage of the Poly-ADP-ribose-polymerase (PARP) suggested that apoptotic pathways may be involved in cell death.

Conclusions: In this study we showed that inhibition of N-linked glycosylation affects ALK phosphorylation and disrupts downstream pro-survival signaling, indicating that inhibition of this post-translational modification may be a promising therapeutic approach. However, as tunicamycin is not a likely candidate for clinical use other approaches to alter N-linked glycosylation need to be explored. Future studies will assess whether the efficacy in inhibiting ALK activity might be enhanced by the combination of ALK specific small molecule and N-linked glycosylation inhibitors.

\footnotetext{
* Correspondence: gianpaolo.tonini@istge.it

${ }^{1}$ Translational Oncopathology, IRCSS A.O.U. San Martino-IST, National Cancer

Research Institute, L.go R. Benzi 10, 16132 Genoa, Italy

Full list of author information is available at the end of the article
} 


\section{Background}

Neuroblastoma (NB) is a pediatric cancer that arises from neural crest cells committed to the adrenal medulla and the sympathetic nervous system. NB accounts for $7-10 \%$ of all childhood cancers and the disease prevalence is about one case in 7,000 live births [1]. Most NB cases are sporadic and may occur either as a localized disease with a favorable prognosis or as a disseminated disease, which, on the contrary, has a worse prognosis in children over 1 year of age and heavily contributes to childhood cancer mortality [1]. In 2008, we participated in discovering that activating germline missense mutations in the catalytic domain of the Anaplastic Lymphoma Kinase (ALK) are a major cause for predisposition to familial NB [2]. In addition, ALK mutations were also found to be somatically acquired in about $8 \%$ of sporadic NB cases, suggesting that ALK is involved in NB carcinogenesis in at least a subset of tumors [2-8]. Moreover, we have investigated ALK expression levels in relationship with the presence of ALK mutations and the clinical outcome of patients, observing that ALK protein expression is significantly upregulated in advanced/metastatic NB, regardless the presence of gene mutations [7].

ALK is an orphan trans-membrane receptor tyrosine kinase (RTK), which belongs to the insulin receptor superfamily of RTKs. The catalytic domain of ALK was originally identified in the $t(2 ; 5)(\mathrm{p} 23 ; \mathrm{q} 35)$ chromosomal translocation that produces the unglycosylated oncogenic fusion protein NPM-ALK, which occurs in Anaplastic Large Cell Lymphoma (ALCL) [9]. ALK is a protein of approximately $180 \mathrm{kDa}$ that consists of 1,620 amino acids and undergoes post-translational modifications such as glycosylation of asparagines, namely, $\mathrm{N}$ linked glycosylation. Indeed, ALK has 16 highly conserved putative sites of N-linked glycosylation in the extra-cellular portion. Consequently, the mature fulllength receptor has a molecular weight of about 220 $\mathrm{kDa}$.

N-linked glycosylation is a highly regulated post-translational modification, which is involved in several biological processes such as protein folding and conformation, oligomerization, sorting, cell-cell interactions, and targeting of proteins to sub- or extra-cellular locations [10,11]. Moreover, several reports have shown a crucial role of N-linked carbohydrates in cell-cycle progression and cell viability [11]. This post-translational modification is initiated upon entry of the polypeptide into the lumen of the endoplasmic reticulum and involves transfer of a carbohydrate moiety to an asparagine $(\mathrm{N})$ residue within a specific amino acid consensus sequence (i.e. NXS/T). The carbohydrate side chain is then processed in the endoplasmic reticulum and Golgi network to produce a mature glycoprotein that is exported through the secretory machinery to the plasma membrane.

Tunicamycin is a specific inhibitor of N-linked glycosylation that blocks the first step of glycoprotein synthesis, thus inhibiting the synthesis of all $\mathrm{N}$-linked glycoproteins. Interestingly, tunicamycin was shown to impair the function of several RTKs such as EGFR, ErbB2, ErbB3, and IGF-IR [12].

We hypothesized that, as demonstrated for other RTKs [12], ALK function may depend on N-linked glycosylation and we investigated the effects of tunicamycin on NB cell lines characterized by different ALK alterations. Our results showed that inhibition of N-linked glycosylation impairs ALK phosphorylation and disrupts downstream pro-survival signaling, as well as cell viability, in NB lines in which ALK is mutated or amplified.

\section{Methods \\ Cell culture}

The following ALK-positive NB cell lines were employed: SH-SY5Y and KELLY (F1174L ALK mutation), UKF-NB3 (R1275Q ALK mutation) and NB1 (ALK amplified). As a control, we included LA1-5S and NB5 NB cell lines that are both ALK-negative, and the ALCL derived cell line SU-DHL1, which harbors the fusion protein NPM-ALK. Human NB cell line SHSY5Y was purchased from the Biological Bank and Cell Factory core facility (National Cancer Research Institute, Genoa, Italy) and the LA1-5S cell line was purchased from the European Collection of Cell Cultures (ECACC). UKF-NB3, NB1, NB5, KELLY and SU-DHL1 cell lines were kindly provided by the Pediatric Oncology Unit (Fondazione IRCCS Istituto Nazionale dei Tumori, Milan, Italy). All cell lines employed in this study have been rechecked for ALK mutations, alterations and expression levels of the protein. LA1-5S were grown in MEM/Ham's F12 1:1 supplemented with nonessential amino acids and 10\% inactivated fetal bovine (Lonza, Basel, Switzerland). All other cell lines were grown in RPMI 1,640 supplemented with $10 \%$ inactivated fetal bovine serum (Lonza). Tunicamycin was purchased from Sigma-Aldrich (St. Louis, MO).

\section{Western blot analysis}

A standard immunoblotting protocol was used to assay protein expression in cell lysates. Cells were washed twice with ice-cold PBS and lysed with 100-200 $\mu \mathrm{l}$ of lysis buffer (Invitrogen, Carlsbad, CA), supplemented with protease and phosphatase inhibitor cocktails and PMSF (phenylmethylsulfonyl fluoride) (Sigma-Aldrich). Lysates were vortexed and incubated on ice for $10 \mathrm{~min}$ twice and then cleared by spinning at $20,000 \times \mathrm{g}$ for 10 
$\min$ at $4^{\circ} \mathrm{C}$. Proteins were separated by $7.5 \%-10 \%$ SDSPAGE gels and immunoblotted according to standard Western blotting procedures using primary antibodies for ALK, AKT, ERK1/2, STAT3 and related phosphoproteins (Cell Signaling, Danvers, MA), and either $\alpha$ tubulin (Cell Signaling) or GAPDH (Abcam, Cambridge, UK) as a control for loading of equal amounts of cell lysates. Goat anti-rabbit and goat anti-mouse IgG HRP conjugated secondary antibodies were from Jackson Immunoresearch Inc (Suffolk, UK) and Santa Cruz Biotechnologies Inc (Santa Cruz, CA), respectively. Blots were developed with Amersham's ECL (GE Healthcare, Waukesha, WI).

Quantification of the western blot bands was performed by ImageJ [13].

\section{MTT assay}

Six replicates of SH-SY5Y, KELLY, UKF-NB3, NB1 and SU-DHL1 (5,000 cells/well) and of LA1-5S (2,500 cells/ well) were plated in 96-well plates with $100 \mu \mathrm{l} \mathrm{RPMI}$ or MEM + Ham's F12 medium additioned with $10 \%$ fetal bovine serum. After $24 \mathrm{~h}$ cells were treated with decreasing concentrations of tunicamycin (500 nM $200 \mathrm{nM}-100 \mathrm{nM}-50 \mathrm{nM}-25 \mathrm{nM}$ ), whereas no treatment was done as positive proliferation control. At $48 \mathrm{~h}$, $25 \mu \mathrm{l}$ of $2 \mathrm{mg} / \mathrm{ml} \mathrm{MTT} \mathrm{(Sigma-Aldrich)} \mathrm{was} \mathrm{added} \mathrm{and}$ plates incubated for $4 \mathrm{~h}$ at $37^{\circ} \mathrm{C}$ and centrifuged $10 \mathrm{~min}$ at $1,500 \mathrm{rpm}$. Finally, $150 \mu \mathrm{l}$ of DMSO were added to each well and absorbance was read at $560 \mathrm{~nm}$.

\section{FACS analysis}

Ethanol fixed cells were incubated with $5 \mu \mathrm{l}$ annexin-V FITC conjugate (Invitrogen) for $10 \mathrm{~min}$ at room temperature in the dark. Thereafter, cells were centrifuged, resuspended in $190 \mu \mathrm{l}$ binding buffer and additioned with $10 \mu \mathrm{l}$ propidium iodide (PI) (Sigma-Aldrich). Cells were analyzed immediately for Annexin-V-FITC and PI binding, using a FACS (fluorescence activated cell sorting) flow cytometer (CyAn ${ }^{\mathrm{TM}}$ Dako). Dot plots and histograms were analyzed by the Dako CyAn - Summit 4.3 software. Annexin-V and PI positive cells were considered apoptotic. Annexin-V and PI negative cells were considered viable.

\section{xCELLigence real-time cell analysis}

SH-SY5Y, KELLY, NB1, LA1-5S (5,000 cells/well), and UKF-NB3 (40,000 cells/well) cells were seeded in EPlates 16 (Roche, Basel, $\mathrm{CH}$ ) in appropriate culture media to a final volume of $200 \mu \mathrm{l}$ and maintained in a $\mathrm{CO}_{2}$ incubator at $37^{\circ} \mathrm{C}$ and $5 \% \mathrm{CO}_{2}$ saturation. Dynamic cell proliferation was monitored in real-time from the time of plating until the end of the experiment. Electrode impedance at the bottom of each well was displayed as cell index, which is a direct measure of cell adhesion and it is a measure that indicates a change in cell behavior due to an increase or decrease in cell number, cell adhesion or cell morphology. Adherent NB cell lines were firstly tested to determine the optimal number of cells to be plated, and cells were allowed to reach a cell index of at least 0.5-1 before treatment. Exponentially growing cells were treated in duplicate with decreasing concentrations of tunicamycin (500 nM, 250 $\mathrm{nM}$ and $125 \mathrm{nM})$. No treatment was used as positive proliferation control. Cell index values were acquired in real-time for at least $48 \mathrm{~h}$ after treatment. Cell index curves were calculated for every tunicamycin treatment and plotted on a graph by Excel.

\section{Results}

Inhibition of N-linked glycosylation impairs ALK phosphorylation and downstream signaling

To investigate the effect that inhibition of N-linked glycosylation could have on ALK phosphorylation and downstream signaling we treated cells with $500 \mathrm{nM}$ tunicamycin, a concentration previously employed in other cell lines of different origin [12], in time course experiments.

Western blot analysis of protein levels showed the accumulation of a hypoglycosylated ALK band (180 $\mathrm{kDa}$ ) and a concomitant decreased of the amount of mature full size receptor $(220 \mathrm{kDa})$ (Figure 1$)$. Interestingly, after $12 \mathrm{~h}$ of treatment, we also observed a marked decrease of ALK phosphorylation in each of the NB cell lines harboring ALK mutation or amplification, and the unphosphorylated status of ALK was still present at $48 \mathrm{~h}$ (Figure 1). On the contrary, tunicamycin treatment performed on the ALCL derived cell line SUDHL1 did not result in any reduction of the phosphorylation levels of NPM-ALK, which lacks N-linked glycosylation consensus sites.

Next, we investigated expression and phosphorylation levels of proteins involved in ALK signaling (i.e. AKT, ERK1/2 and STAT3). Tunicamycin treatment did not affect AKT, ERK1/2 and STAT3 expression (Figure 1), whereas their phosphorylation levels decreased considerably within $48 \mathrm{~h}$. Noteworthy, we detected an evident rephosphorylation of STAT3 in SH-SY5Y cells after 48 $\mathrm{h}$ of tunicamycin treatment (Figure 1). As tunicamycin impairs N-linked glycosylation of all newly synthesized glycoproteins, we also treated LA1-5S, NB5 and SUDHL1 cell lines, which do not rely on native ALK as an oncogenic driver. Intriguingly, tunicamycin did not affect phosphorylation levels of ALK downstream effectors in these control cell lines, apart from a temporary reduction of phospho-ERK1/2 levels in LA1-5S cells (Figure 1). 

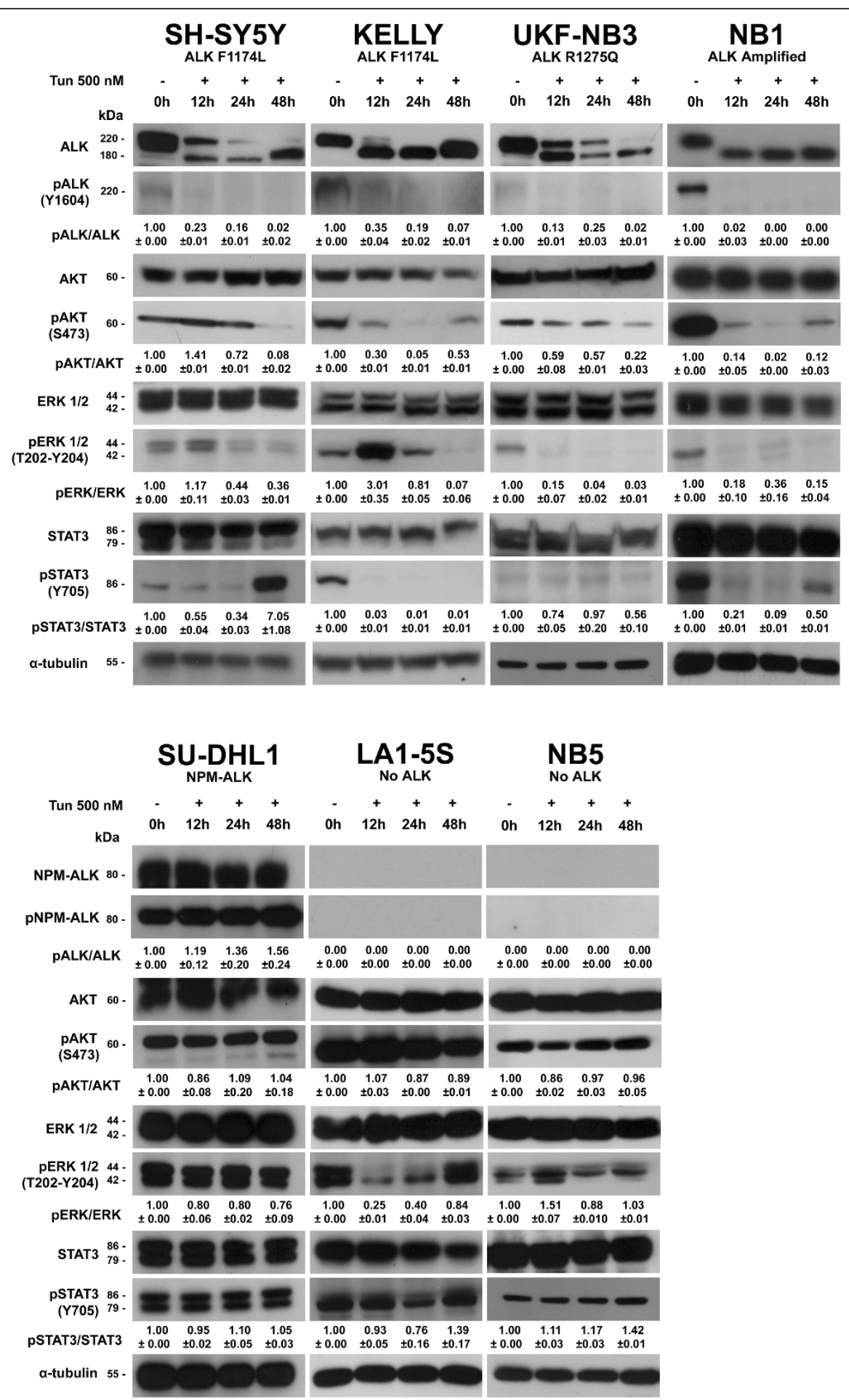

Figure 1 Effects of tunicamycin on ALK expression and phosphorylation and on downstream signaling proteins. Cells were exposed to $500 \mathrm{nM}$ tunicamycin and treated in time course experiments. Expression levels of ALK and phosphorylation of ALK (Tyr1604), AKT (Ser473), ERK1/ 2 (Thr202/Tyr204), STAT3 (Tyr705) and NPM-ALK (Tyr664) were detected in total cell lysates by Western blot, using corresponding antibodies. $\alpha$ tubulin was used as sample loading control. Quantification of western blot bands was performed by using ImageJ software [13]. Quantification values for each band were firstly normalized to corresponding values of $\alpha$-tubulin. Then, normalized values for each time were compared to the corresponding band of the untreated sample (0 hours). Finally the ratio between the phosphorylated form of the proteins and its unphosphorylated counterpart for each experimental time was calculated and reported with standard deviations. 


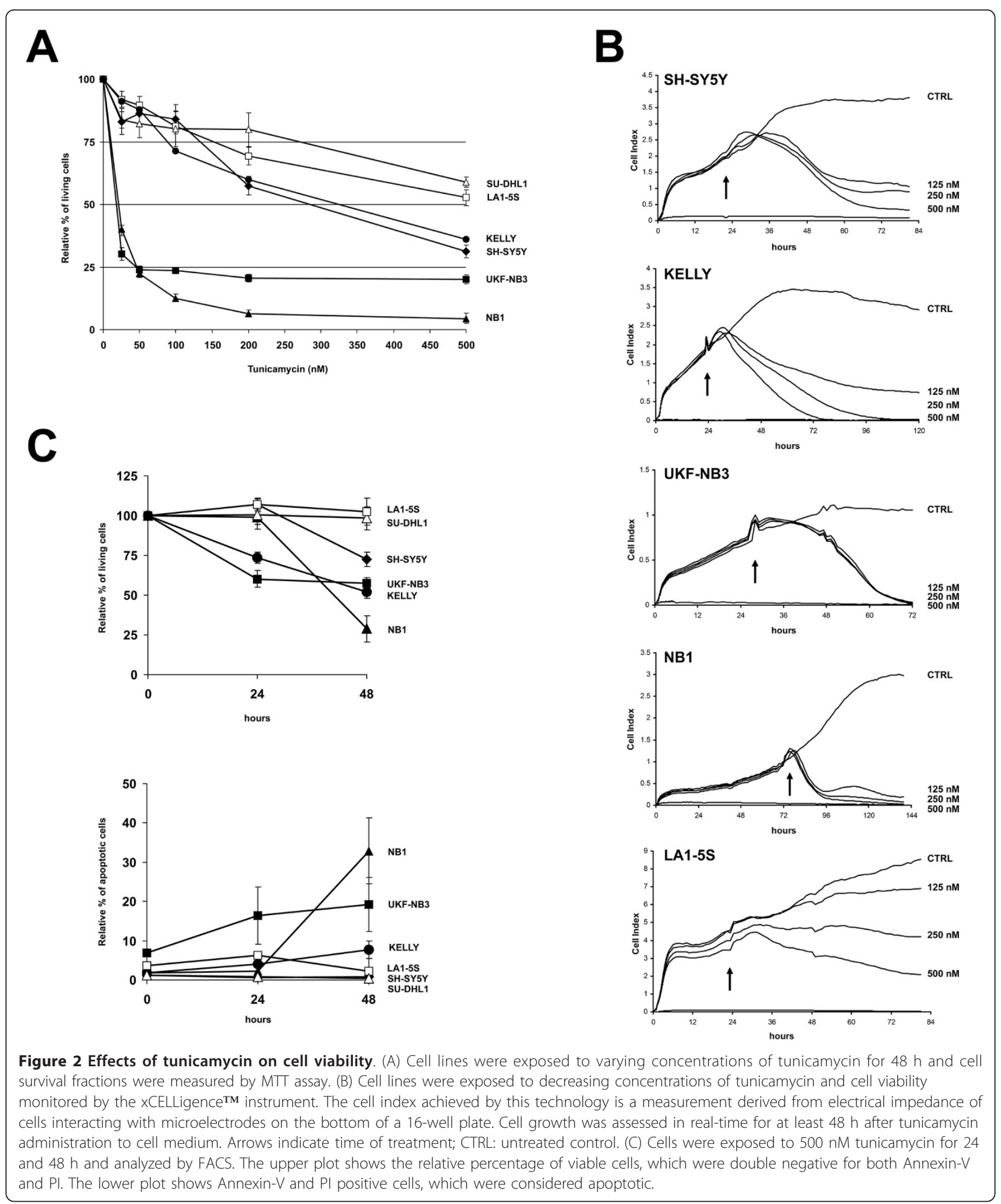


Inhibition of $\mathrm{N}$-linked glycosylation affects cell viability of ALK-driven NB cell lines

To evaluate the effect of inhibition of N-linked glycosylation on cell survival, cells were cultured for $48 \mathrm{~h}$ with increasing concentrations of tunicamycin, ranging from $25 \mathrm{nM}$ to $500 \mathrm{nM}$, and cell viability was tested by MTT assay (Figure 2A). Cell survival fractions were evaluated in comparison to control cell survival. We observed a profound inhibition of cell growth in both UKF-NB3 and NB1 cells that showed an $\mathrm{IC}_{50}$ of about $20 \mathrm{nM}$, whereas SH-SY5Y and KELLY cells were more resistant, showing an $\mathrm{IC}_{50}$ of $283 \mathrm{nM}$ and $325 \mathrm{nM}$, respectively (Figure 2A). On the other hand, both ALK-negative LA1-5S cells and NPM-ALK-positive SU-DHL1 cells were definitely resistant to tunicamycin, showing an $\mathrm{IC}_{50}>500 \mathrm{nM}$ (Figure 2A).

Cell viability was also analyzed by $\mathrm{xCELLigence}{ }^{\mathrm{TM}}$ instrument (Roche), which monitors cell adhesion in real-time (Figure 2B). As a result, cell index of each NB cell lines harboring ALK mutation or amplification was extensively impaired, whereas cell index of LA1-5S cells resulted minimally affected (Figure $2 \mathrm{~B}$ ). Noteworthy, in contrast to MTT results, we observed a marked decrease of cell growth also for SH-SY5Y and KELLY cells. In fact, as for the other ALK-positive cell lines, also SHSY5Y and KELLY looked suffering at observation and most of the cells were detached from the bottom of the flask after $48 \mathrm{~h}$ treatment with $500 \mathrm{nM}$ tunicamycin (Figure 3).

Finally, cell lines were analyzed by flow cytometry after double staining with annexin-V and PI. After $48 \mathrm{~h}$ of treatment with $500 \mathrm{nM}$ tunicamycin, SH-SY5Y, KELLY, UKF-NB3, and NB1 cells showed a cell viability reduction of $27 \%, 48 \%, 42 \%$, and $71 \%$, respectively, whereas no significant variation was observed in both LA1-5S and SU-DHL1 cells (Figure 2C). Moreover, about $6 \%$ of KELLY, $13 \%$ of UKF-NB3 and 31\% of NB1 cells displayed double staining to both annexin- $\mathrm{V}$ and $\mathrm{PI}$, and were considered apoptotic (Figure 2C).

Since both NB1 and UKF-NB3 showed an $\mathrm{IC}_{50}$ greatly lower than the tunicamycin concentration used in the first set of experiments, we employed $50 \mathrm{nM}$ tunicamycin in ALK-positive NB cell lines. As a result, we observed similar effects on ALK and its effectors in NB1 and UKF-NB3 (Figure 4) but not in SH-SY5Y and KELLY cells, which in fact were more resistant as detected by MTT assay.

\section{Activation of apoptotic pathways in tunicamycin treated cells}

To investigate the involvement of apoptotic pathways after inhibition of $\mathrm{N}$-linked glycosylation we analyzed the following apoptotic proteins: caspase-3, caspase-8, caspase-9, caspase-12 and the Poly-ADP-ribose-

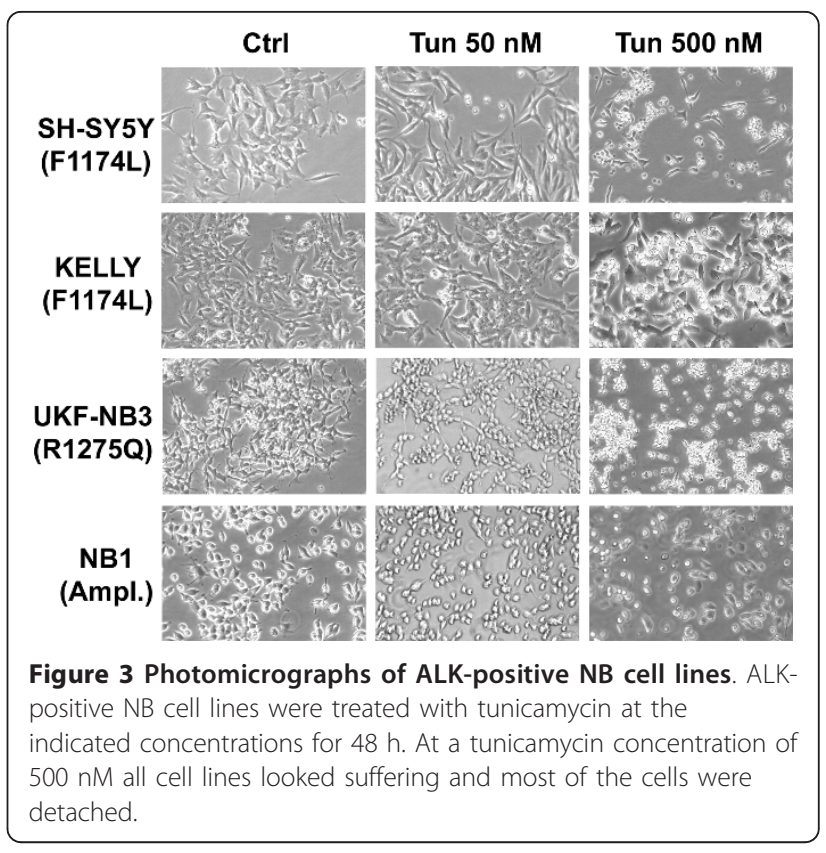

polymerase (PARP). Cells were exposed to $500 \mathrm{nM}$ tunicamycin for $48 \mathrm{~h}$. We did not detect cleavage fragments in any of the analyzed caspases. On the contrary, we observed cleavage fragments of PARP in all ALK-positive NB cell lines but not in control cell lines (Figure $5 \mathrm{~A})$. Cleavage fragments of PARP were still present after treatment of SH-SY5Y and NB1 cells with both $20 \mu \mathrm{M}$ of the pan-caspase inhibitor BOC-D-fmk (Calbiochem, Gibbstown, NJ) and $500 \mathrm{nM}$ of tunicamycin, indicating a caspase independent activation of PARP (Figure 5B).

\section{Discussion}

In the present study, we addressed the question of whether ALK function may depend on N-linked glycosylation. Inhibition of ALK activity in NB cell lines has already been approached by using specific small molecule ALK inhibitors, such as PF-2341066 [3], NVPTAE684 [4] and CEP14083/CEP14513 [7], and more recently by RNA interference molecules [14]. Particularly, NB cells harboring either R1275Q mutation or ALK amplification showed sensitivity to PF-02341066 and these results were also confirmed in xenografts (Wood AC et al., ASCO Annual Meeting, 2009). However, NB cell lines with both F1174L ALK mutations and wild type ALK were more resistant. Furthermore, two secondary mutations in the kinase domain of the fusion protein EML4-ALK were discovered in nonsmall-cell lung cancer in tumor cells isolated from a patient during the relapse phase of treatment with the ALK inhibitor PF-02341066 [15]. Each mutation developed independently in subclones of the tumor and conferred marked resistance to two different ALK 


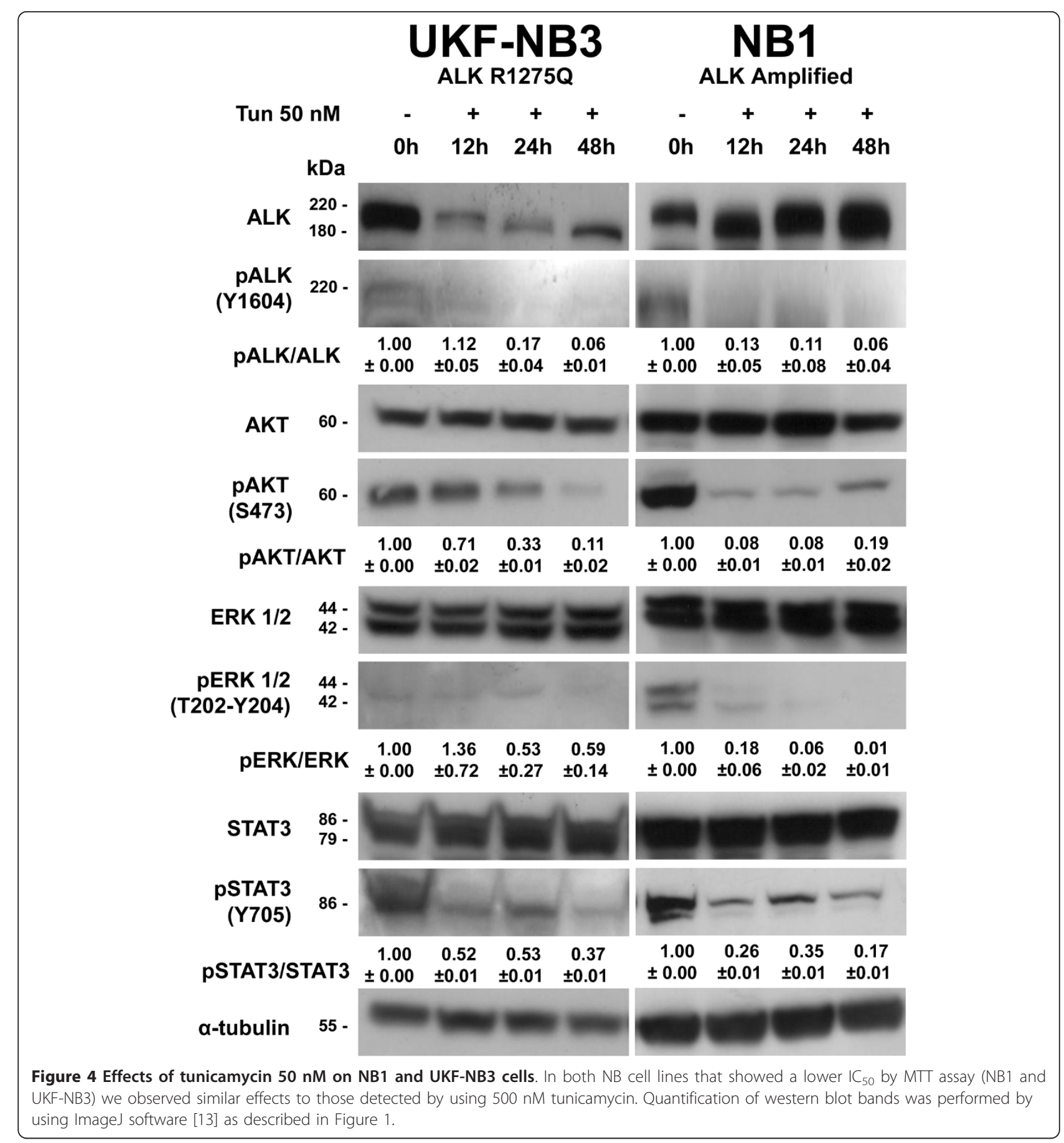

inhibitors, PF-02341066 and a 2,4-pyrimidinediamine derivative (PDD) [15]. Although small molecule inhibitors are a class of anticancer compounds that have shown promising clinical activity, resistance phenomena may occur, in part due to either primary and secondary mutations or to the plasticity of regulation of downstream signaling pathways. Hence, great efforts are now aimed at finding efficient ALK inhibitors, at least for the more frequent ALK alterations.

In 2008, Contessa and colleagues reported that inhibition of N-linked glycosylation reduced RTK (i.e. EGFR, ErbB2, ErbB3 and IGF-IR) signaling through AKT and radiosensitized tumor cells in glioma and pancreatic adenocarcinoma cell lines. In comparison, experiments 


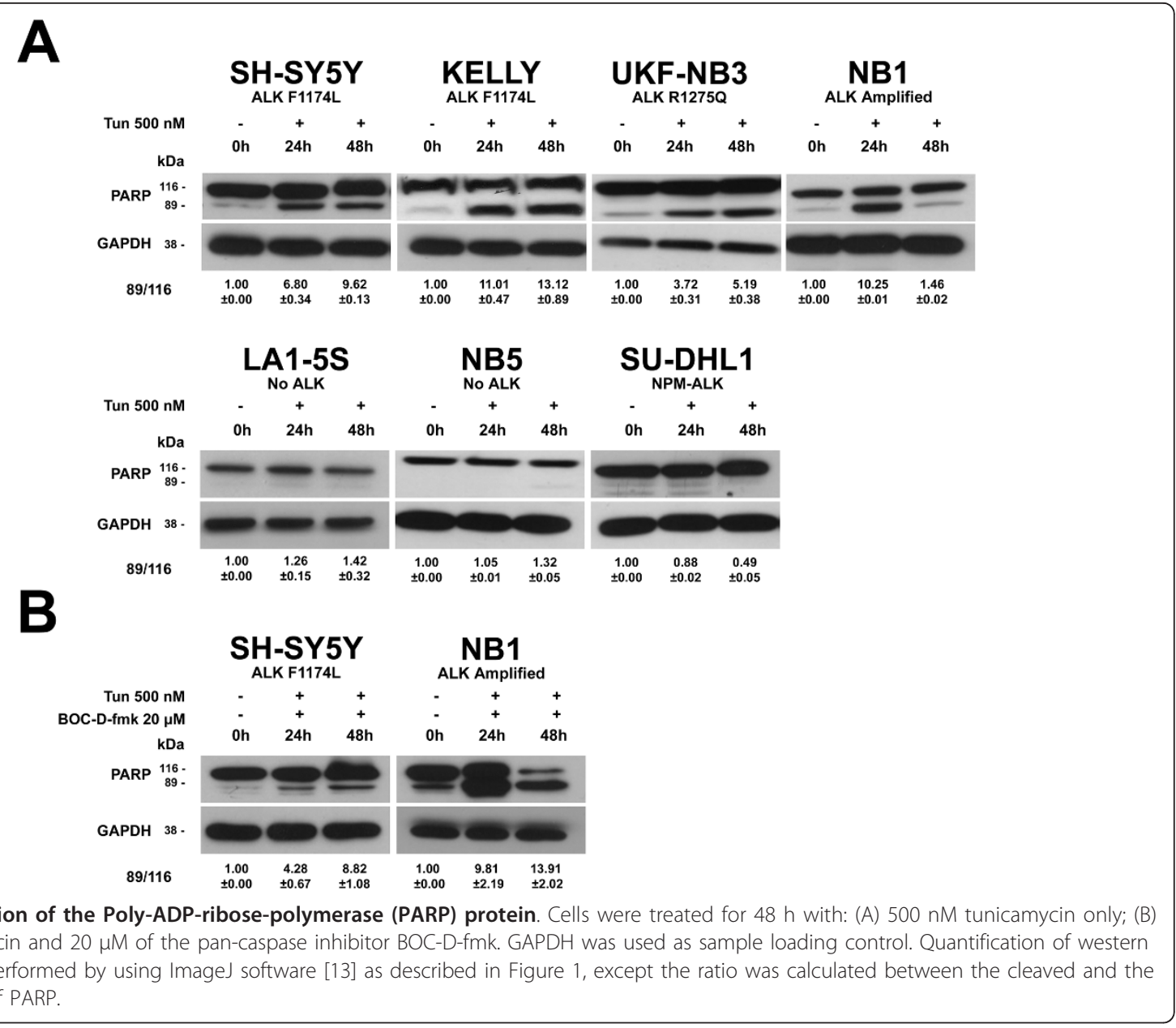

carried out in nontransformed fibroblastic cells showed neither a reduction in RTK dependent signaling nor an enhancement in radiosensitivity [12]. Afterwards, they also provided evidences that disruption of N-linked glycosylation could reduce RTK signaling in vivo and increase radiosensitivity of gliomas, suggesting that targeting $\mathrm{N}$-linked glycosylation may combine favorably with other classes of EGFR inhibitors to reduce both oncogenic signaling and the mechanisms of therapeutic resistance [16]. Moreover, tunicamycin was showed to enhance the susceptibility of lung cancer cells and sensitize resistant cell lines to Erlotinib [17].

Similarly, our results indicated that N-linked glycosylation has a pivotal role also in ALK activation, as described for EGFR and other RTKs [12]. Tunicamycin has broad, non-specific effects and acts on all N-linked glycoproteins. Nevertheless, we specifically investigated the effects of tunicamycin on NB cell lines that are dependent on ALK for survival. Here, we showed that inhibition of $\mathrm{N}$-linked glycosylation led to reduced or undetectable phosphorylation of ALK. Moreover, we also observed that pro-survival ALK effectors are inactivated only in NB cell lines addicted to native ALK receptor as an oncogenic driver. On the contrary, ALKnegative NB cell lines did not show evident impairment of signaling molecules such as AKT, ERK1/2 and STAT3. Noteworthy, although tunicamycin has broad effects, we did not observe any variation in the phosphorylation levels of ALK effectors in SU-DHL1 cells, which depend on the unglycosylated cytoplasmatic protein NPM-ALK. The reactivation of STAT3 observed in SH-SY5Y cells may be explained by considering a possible redundancy of cellular signaling networks. Indeed, multiple RTKs may be coactivated in tumors and redundant inputs drive and maintain downstream signaling $[18,19]$, although other possibilities should not be ruled out.

Inhibition of N-linked glycosylation also impaired cell proliferation only of ALK-positive NB cells. Particularly, UKF-NB3 and NB1 cells showed high sensitivity to tunicamycin by MTT assay, whereas SH-SY5Y and KELLY cells were less sensitive. However, cell viability assays carried out by flow cytometry and real-time monitoring of cell adhesion showed a marked cell growth reduction 
for all ALK-positive NB cell lines, including SH-SY5Y and KELLY. Finally, the cleavage of the Poly-ADPribose-polymerase (PARP) was observed only in ALKpositive NB cell lines, suggesting that apoptotic pathways may be involved in cell death.

\section{Conclusions}

In this study we showed that the inhibition of N-linked glycosylation has the capability to affect ALK phosphorylation and disrupt pro-survival signaling associated to ALK, indicating that inhibition of this post-translational modification may be a promising therapeutic approach for ALK-depending NB patients. Moreover, the contributions of coexpressed growth factor receptors to developing resistance to therapeutic RTK inhibitors have been shown, confirming that inhibition of multiple RTKs may be of therapeutic benefit. Thus, as tunicamycin acts on all N-linked glycoproteins it is plausible to suppose that, differently from specific small molecule inhibitors, disruption of $\mathrm{N}$-linked glycosylation should reduce a reliance on networks of RTKs that may overlap in effects with ALK.

Since tunicamycin is not a likely candidate for clinical use our results are to be taken as a proof-of-concept and other approaches to alter N-linked glycosylation need to be explored. Future studies will assess whether the efficacy in inhibiting ALK activity might be enhanced by the combination of ALK specific small molecule and $\mathrm{N}$-linked glycosylation inhibitors.

\begin{abstract}
Acknowledgements
We would like to thank Dr. Francesca Tosetti (National Cancer Research Institute, Genoa, Italy) for supplying us with caspases and PARP antibodies, and the pan-caspase inhibitor. We would also like to thank Prof. Michela Tonetti and Dr. Cinzia Bernardi (Department of Experimental Medicine, University of Genoa, Italy), and Dr. Laura Paleari (Italian Neuroblastoma Foundation and National Cancer Research Institute, Genoa, Italy) for helpful discussion of results.

This work has been supported by Italian Neuroblastoma Foundation, Fondazione CARIGE and Italian Association for Cancer Research (AIRC). LP is supported and granted by Fondazione Umberto Veronesi (FUV), Milano.

\section{Author details \\ ${ }^{1}$ Translational Oncopathology, IRCSS A.O.U. San Martino-IST, National Cancer Research Institute, L.go R. Benzi 10, 16132 Genoa, Italy. ${ }^{2}$ Department of Experimental Medicine, University of Genoa, Via L. Battista Alberti 2, 16132 Genoa, Italy. ${ }^{3}$ Pediatric Oncology Unit, Fondazione IRCCS Istituto Nazionale dei Tumori, Via Venezian 1, 20133 Milano, Italy. ${ }^{4}$ Italian Neuroblastoma Foundation, L.go Gaslini 5, 16147 Genoa, Italy. ${ }^{5}$ Department of Medical Pharmacology, University of Milano, Via Vanvitelli 32, 20129 Milano, Italy.}

\section{Authors' contributions}

FDG designed and performed experiments, interpreted results. MDM performed experiments. LP performed experiments and critically revised the manuscript. RL critically revised the manuscript. GPT critically revised the manuscript. LL conceived of the study, designed experiments, interpreted results, drafted and critically revised the manuscript. All authors read and approved the final manuscript.

\section{Competing interests}

The authors declare that they have no competing interests.
Received: 21 June 2011 Accepted: 22 December 2011

Published: 22 December 2011

\section{References}

1. Brodeur GM: Neuroblastoma: biological insights into a clinical enigma. Nat Rev Cancer 2003, 3:203-216.

2. Mossé YP, Laudenslager M, Longo L, Cole KA, Wood A, Attiyeh EF, Laquaglia MJ, Sennett R, Lynch JE, Perri P, Laureys G, Speleman F, Kim C, Hou C, Hakonarson H, Torkamani A, Schork NJ, Brodeur GM, Tonini GP, Rappaport E, Devoto M, Maris JM: Identification of ALK as a major familial neuroblastoma predisposition gene. Nature 2008, 455:930-935.

3. Janoueix-Lerosey I, Lequin $D$, Brugières $L$, Ribeiro $A$, de Pontual $L$, Combaret V, Raynal V, Puisieux A, Schleiermacher G, Pierron G, ValteauCouanet D, Frebourg T, Michon J, Lyonnet S, Amiel J, Delattre O: Somatic and germline activating mutations of the ALK kinase receptor in neuroblastoma. Nature 2008, 455:967-970.

4. George RE, Sanda T, Hanna M, Fröhling S, Luther W, Zhang J, Ahn Y, Zhou W, London WB, McGrady P, Xue L, Zozulya S, Gregor VE, Webb TR, Gray NS, Gilliland DG, Diller L, Greulich H, Morris SW, Meyerson M, Look AT: Activating mutations in ALK provide a therapeutic target in neuroblastoma. Nature 2008, 455:975-978.

5. Chen Y, Takita J, Choi YL, Kato M, Ohira M, Sanada M, Wang L, Soda M, Kikuchi A, Igarashi T, Nakagawara A, Hayashi Y, Mano H, Ogawa S: Oncogenic mutations of ALK kinase in neuroblastoma. Nature 2008, 455:971-974.

6. Carén $H$, Abel $F$, Kogner $P$, Martinsson $T$ : High incidence of DNA mutations and gene amplifications of the ALK gene in advanced sporadic neuroblastoma tumours. Biochem J 2008, 416:153-159.

7. Passoni L, Longo L, Collini P, Coluccia AM, Bozzi F, Podda M, Gregorio A, Gambini C, Garaventa A, Pistoia V, Del Grosso F, Tonini GP, Cheng M, Gambacorti-Passerini C, Anichini A, Fossati-Bellani F, Di Nicola M, Luksch R: Mutation-independent anaplastic lymphoma kinase overexpression in poor prognosis neuroblastoma patients. Cancer Res 2009, 69:7338-7346.

8. De Brouwer S, De Preter K, Kumps C, Zabrocki P, Porcu M, Westerhout EM, Lakeman A, Vandesompele J, Hoebeeck J, Van Maerken T, De Paepe A, Laureys G, Schulte JH, Schramm A, Van Den Broecke C, Vermeulen J, Van Roy N, Beiske K, Renard M, Noguera R, Delattre O, Janoueix-Lerosey I, Kogner P, Martinsson T, Nakagawara A, Ohira M, Caron H, Eggert A, Cools J, Versteeg $R$, Speleman F: Meta-analysis of neuroblastomas reveals a skewed ALK mutation spectrum in tumors with MYCN amplification. Clin Cancer Res 2010, 16(17):4353-4362.

9. Morris SW, Kirstein MN, Valentine MB, Dittmer KG, Shapiro DN, Saltman DL, Look AT: Fusion of a kinase gene, ALK, to a nucleolar protein gene, NPM, in non-Hodgkin's lymphoma. Science 1994, 263:1281-1284.

10. Gupta G, Sinha S, Mitra N, Surolia A: Probing into the role of conserved Nglycosylation sites in the Tyrosinase glycoprotein family. Glycoconj J 2009, 26:691-695.

11. Helenius A, Aebi M: Intracellular functions of $\mathrm{N}$-linked glycans. Science 2001, 291:2364-2369.

12. Contessa JN, Bhojani MS, Freeze HH, Rehemtulla A, Lawrence TS: Inhibition of $\mathrm{N}$-linked glycosylation disrupts receptor tyrosine kinase signaling in tumor cells. Cancer Res 2008, 68:3803-3809.

13. Abramoff MD, Magalhaes PJ, Ram SJ: Image Processing with ImageJ. Biophotonics International 2004, 11:36-42.

14. Di Paolo D, Brignole C, Pastorino F, Carosio R, Zorzoli A, Rossi M, Loi M, Pagnan G, Emionite L, Cilli M, Bruno S, Chiarle R, Allen TM, Ponzoni M, Perri P: Neuroblastoma-targeted nanoparticles entrapping siRNA specifically knockdown ALK. Mol Ther 2011, 19:1131-1140.

15. Choi YL, Soda M, Yamashita Y, Ueno T, Takashima J, Nakajima T, Yatabe Y, Takeuchi K, Hamada T, Haruta H, Ishikawa Y, Kimura H, Mitsudomi T, Tanio Y, Mano H, ALK Lung Cancer Study Group: EML4-ALK mutations in lung cancer that confer resistance to ALK inhibitors. N Engl J Med 2010, 363:1734-1739.

16. Contessa JN, Bhojani MS, Freeze HH, Ross BD, Rehemtulla A, Lawrence TS: Molecular imaging of $\mathrm{N}$-linked glycosylation suggests glycan biosynthesis is a novel target for cancer therapy. Clin Cancer Res 2010, 16:3205-3214.

17. Ling YH, Li T, Perez-Soler R, Haigentz M Jr: Activation of ER stress and inhibition of EGFR N-glycosylation by tunicamycin enhances susceptibility of human non-small cell lung cancer cells to erlotinib. Cancer Chemother Pharmacol 2009, 64:539-548. 
18. Stommel JM, Kimmelman AC, Ying $H$, Nabioullin R, Ponugoti $A H$, Wiedemeyer R, Stegh AH, Bradner JE, Ligon KL, Brennan C, Chin L, DePinho RA: Coactivation of receptor tyrosine kinases affects the response of tumor cells to targeted therapies. Science 2007, 318:287-290.

19. Wang S, Pashtan I, Tsutsumi S, Xu W, Neckers L: Cancer cells harboring MET gene amplification activate alternative signaling pathways to escape MET inhibition but remain sensitive to Hsp90 inhibitors. Cell Cycle 2009, 8:2050-2056

Pre-publication history

The pre-publication history for this paper can be accessed here:

http://www.biomedcentral.com/1471-2407/11/525/prepub

doi:10.1186/1471-2407-11-525

Cite this article as: Del Grosso et al: Inhibition of N-linked glycosylation impairs ALK phosphorylation and disrupts pro-survival signaling in neuroblastoma cell lines. BMC Cancer 2011 11:525.

Submit your next manuscript to BioMed Central and take full advantage of:

- Convenient online submission

- Thorough peer review

- No space constraints or color figure charges

- Immediate publication on acceptance

- Inclusion in PubMed, CAS, Scopus and Google Scholar

- Research which is freely available for redistribution

Submit your manuscript at www.biomedcentral.com/submit 